04.2

\title{
Измерение мощности радиационных потерь и эффективного заряда плазмы на токамаке Глобус-М2
}

\author{
(C) Е.А. Тюхменева ${ }^{1}$, Н.Н. Бахарев ${ }^{1}$, В.И. Варфоломеев ${ }^{1}$, В.К. Гусев ${ }^{1}$, Н.С. Жильцов ${ }^{1}$, Е.О. Киселев ${ }^{1}$, \\ Г.С. Курскиев ${ }^{1}$, В.Б. Минаев ${ }^{1}$, Ю.В. Петров ${ }^{1}$, Н.В. Сахаров ${ }^{1}$, А.Д. Сладкомедова ${ }^{2}$, А.Ю. Тельнова ${ }^{1}$, \\ С.Ю. Толстяков ${ }^{1}$, П.Б. Щеголев ${ }^{1}$
}

${ }^{1}$ Физико-технический институт им. А.Ф. Иофффе РАН, Санкт-Петербург, Россия

${ }^{2}$ York Plasma Institute, University of York, York, U.K.

E-mail: tuxmeneva@gmail.com

Поступило в Редакцию 17 июля 2020г.

В окончательной редакции 9 сентября 2020 г.

Принято к публикации 26 сентября 2020 г.

Представлены результаты измерения мощности радиационных потерь $P_{\text {rad }}$ и эффективного заряда плазмы $Z_{\text {eff }}$ в условиях магнитного поля до 0.7 Т и тока по плазме до $300 \mathrm{kA}$ для широкого диапазона электронной плотности, впервые полученные на токамаке Глобус-М2. Анализ результатов показал, что в токамаке Глобус-М2 доля радиационных потерь относительно вкладываемой мощности снизилась по сравнению с полученной на установке Глобус-М, а измеренные профили мощности $P_{\text {rad }}$ имели сильный провал в центре. Кроме того, при повышении плотности электронов наблюдается уменьшение значений $Z_{e f f}$.

Ключевые слова: термоядерный синтез, диагностика плазмы, эффективный заряд плазмы, радиационные потери.

DOI: 10.21883/PJTF.2021.02.50537.18471

На модернизированном токамаке Глобус-М2 (УНУ „Сферический токамак Глобус-М, входит в состав ФЦКП „Материаловедение и диагностика в передовых технологиях“, уникальный идентификатр проекта RFMEF162119X0021) [1-3] первые значительные результаты с использованием всех важнейших диагностик были получены во время экспериментальной кампании в 2019 г. [4]. Измерения проводились при следующих параметрах: тороидальное магнитное поле $B_{T}$ до $0.7 \mathrm{~T}$, ток по плазме $I_{p}$ до $300 \mathrm{kA}$, электронная плотность $n_{e}$ до $10^{20} \mathrm{~m}^{-3}$, температура электронов $T_{e}$ до $1.5 \mathrm{keV}$, температура ионов $T_{i}$ около $1 \mathrm{keV}$. В настоящей работе представлены результаты первых измерений мощности радиационных потерь $P_{\text {rad }}$ и эффективного заряда плазмы $Z_{e f f}$. Пространственное распределение мощности радиационных потерь $P_{\text {rad }}$ и величина эффективного заряда $Z_{e f f}$ дают важную информацию о поступлении и переносе примесей в плазме токамака.

Первые эксперименты по нагреву и удержанию плазмы в токамаке Глобус-М2 были проведены при $B_{T}=0.7 \mathrm{~T}$ и $I_{p}=300 \mathrm{kA}$. Основной характеристикой степени загрязненности плазмы примесными элементами является величина эффективного заряда $Z_{e f f}$, который определяется формулой [5]:

$$
Z_{e f f} \stackrel{\text { def }}{=} \frac{\Sigma_{i} n_{i} Z_{i}^{2}}{\Sigma_{i} n_{i} Z_{i}}
$$

где $n_{i}$ - концентрация ионного компонента плазмы с зарядом $Z_{i}$.

Для измерения эффективного заряда плазмы на токамаке Глобус-М2 была создана диагностика [6], основанная на измерении мощности тормозного излучения
$P_{b r}\left(Z_{e f f}\right)$ в ближнем ИК-диапазоне, где отсутствует интенсивное линейчатое излучение, а рекомбинационное излучение в условиях измерений пренебрежимо мало. Кроме того, использовались данные диагностики томсоновского рассеяния (ТР) [7].

Регистрация тормозного излучения проводится с помощью фильтрового монохроматора, состоящего из объектива, формирующего область наблюдения в виде квазицилиндрического объема диаметром $d=3 \mathrm{~cm}$, интерференционного фильтра для выделения необходимого спектрального интервала и приемника - лавинного фотодиода Hamamatsu S11519-30, оснащенного малошумящим усилителем. Хорда наблюдения, вдоль которой регистрируется $P_{b r}$, проходящая в экваториальном сечении камеры токамака (рис. $1, a)$, идентична хорде зондирования диагностики ТР [7], что в условиях тороидальной симметрии позволяет наиболее точно определять распределения $T_{e}$ и $n_{e}$ в объеме наблюдения. Поскольку линия наблюдения направлена в область патрубка на противоположной стенке, вклад отраженного излучения оказывается значительно меньше, чем в том случае, когда в поле зрения оказывается графитовая поверхность.

По измеренному значению $P_{b r}[\mathrm{~W}]$ и зависимостям $T_{e}(R)[\mathrm{eV}]$ и $n_{e}(R)\left[\mathrm{m}^{-3}\right]$ от большого радиуса $R[\mathrm{~m}]$ в предположении $\left\langle Z_{e f f}\right\rangle(R)=$ const в области наблюдения рассчитывается средний по объему наблюдения эффективный заряд плазмы $\left\langle Z_{e f f}\right\rangle[6]$ :

$$
\left\langle Z_{e f f}\right\rangle=\frac{P_{b r}}{1.5 \cdot 10^{-23}\left\langle g_{f f}\right\rangle \int_{V} \int_{\Delta \lambda} \Omega \frac{n_{e}^{2}}{\lambda^{2} \sqrt{T_{e}}} \exp \left(-\frac{h c}{\lambda T_{e}}\right) d V d \lambda} .
$$



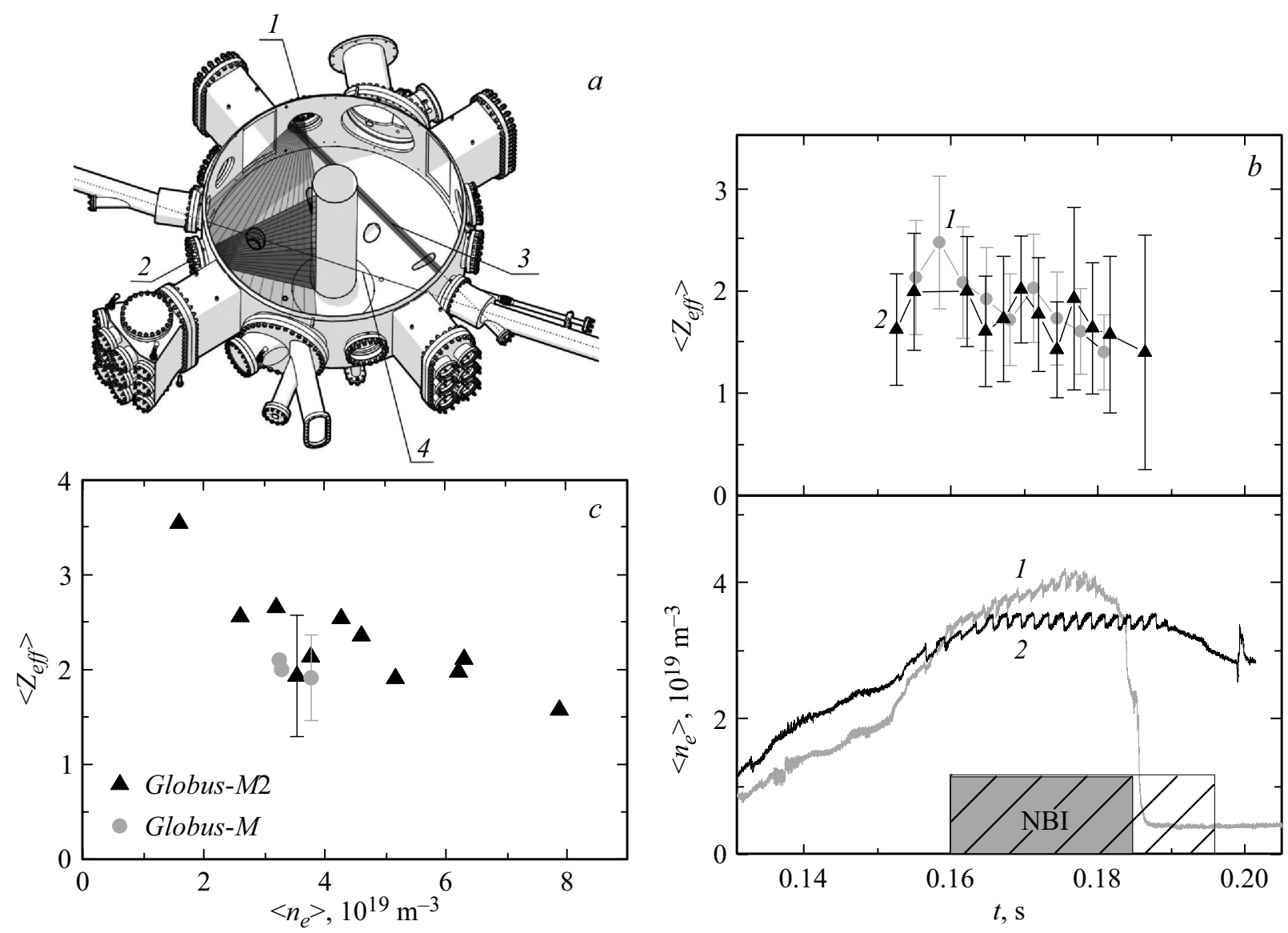

Рис. 1. $a-$ геометрия диагностик $Z_{e f f}[3]$ и $P_{\text {rad }}[8]$ на токамаке Глобус-М2: $1-$ фотодиодная матрица $16 \times 16,2-$ фотодиодная линейка $1 \times 24,3$ - линия наблюдения диагностики $Z_{e f f}, 4$ - лазер диагностики ТP; $b$ - эволюция $\left\langle Z_{e f f}(t)\right\rangle$ и средней плотности электронов $\left\langle n_{e}(t)\right\rangle$ в разрядах \#37069 (Глобус-М) (1) и \#38098 (Глобус-М2) (2); $c$ - зависимость эффективного заряда плазмы $\left\langle Z_{\text {eff }}\right\rangle$ от плотности электронов $\left\langle n_{e}\right\rangle$.

Интегрирование производится по длине волны $\lambda[\mathrm{nm}]$ на спектральном промежутке $\Delta \lambda[\mathrm{nm}]$ и по объему $V\left[\mathrm{~m}^{3}\right]$, в котором регистрируется тормозное излучение. Телесный угол $\Omega[\mathrm{sr}]$, в котором происходит сбор излучения, зависит от расстояния $l(R)[\mathrm{m}]$ до монохроматора, так как объем наблюдения квазицилиндрический.

Спектральный интервал 1020-1041 nm был выбран на основе данных, полученных на токамаке Глобус-М2 с помощью обзорного спектрометра AvaSpec-2048, peгистрирующего спектры излучения плазмы в диапазоне $200-1100 \mathrm{~nm}$.

На рис. $1, b$ приведено сравнение временно́й эволюции $\left\langle Z_{e f f}(t)\right\rangle$ и средней плотности электронов $\left\langle n_{e}(t)\right\rangle$ в разрядах токамаков Глобус-М и ГлобусM2. Разряды были выбраны таким образом, чтобы электронные плотности были одинаковыми. Параметры выбранных разрядов токамаков Глобус-М/Глобус-М2 (\#37069/\#38098 соответственно) были следующими: ток по плазме $I_{p}=180 \mathrm{kA} / 300 \mathrm{kA}$, тороидальное магнитное поле $B_{T}=0.4 \mathrm{~T} / 0.7 \mathrm{~T}$, мощность дополнительного нагрева нейтральным пучком $P_{\mathrm{NBI}}=650 \mathrm{~kW} / 850 \mathrm{~kW}$, энергия частиц пучка $E_{\mathrm{NBI}}=26 \mathrm{keV} / 28 \mathrm{keV}$.
Погрешность определения эффективного заряда в основном зависит от точности измерения плотности электронов $n_{e}$ и температуры $T_{e}$ с помощью диагностики TP [4], а также от погрешности измерения мощности тормозного излучения, которая обусловлена преимущественно неопределенностью вклада излучения, отраженного от стенки, ошибкой определения коэффициента усиления лавинного фотодиода и спектральной характеристики фильтра. В связи с особенностью геометрии регистрации $P_{b r}$ на токамаке Глобус-М2 при расчете погрешности измерения $\left\langle Z_{e f f}\right\rangle$ можно пренебречь вкладом излучения, отраженного от стенки.

Измерения $\left\langle Z_{e f f}\right\rangle$ проводились в широком диапазоне плотностей электронов $n_{e} \approx(0.5-8.0) \cdot 10^{19} \mathrm{~m}^{-3}$. В результате получена зависимость $\left\langle Z_{e f f}\right\rangle$ от электронной плотности $n_{e}$, которая представлена на рис. $1, c$. Для разрядов с пучком значение $\left\langle Z_{\text {eff }}\right\rangle$ взято на $18 \mathrm{~ms}$ от начала инжекции, для омических разрядов - в момент времени с максимальной плотностью $n_{e}$ в стационарной стадии разряда. Результаты измерения демонстрируют уменьшение величины $\left\langle Z_{e f f}\right\rangle$ с увеличением электронной плотности $n_{e}$, что соответствует уменьшению доли содержания примеси при напуске рабочего газа. Такая 

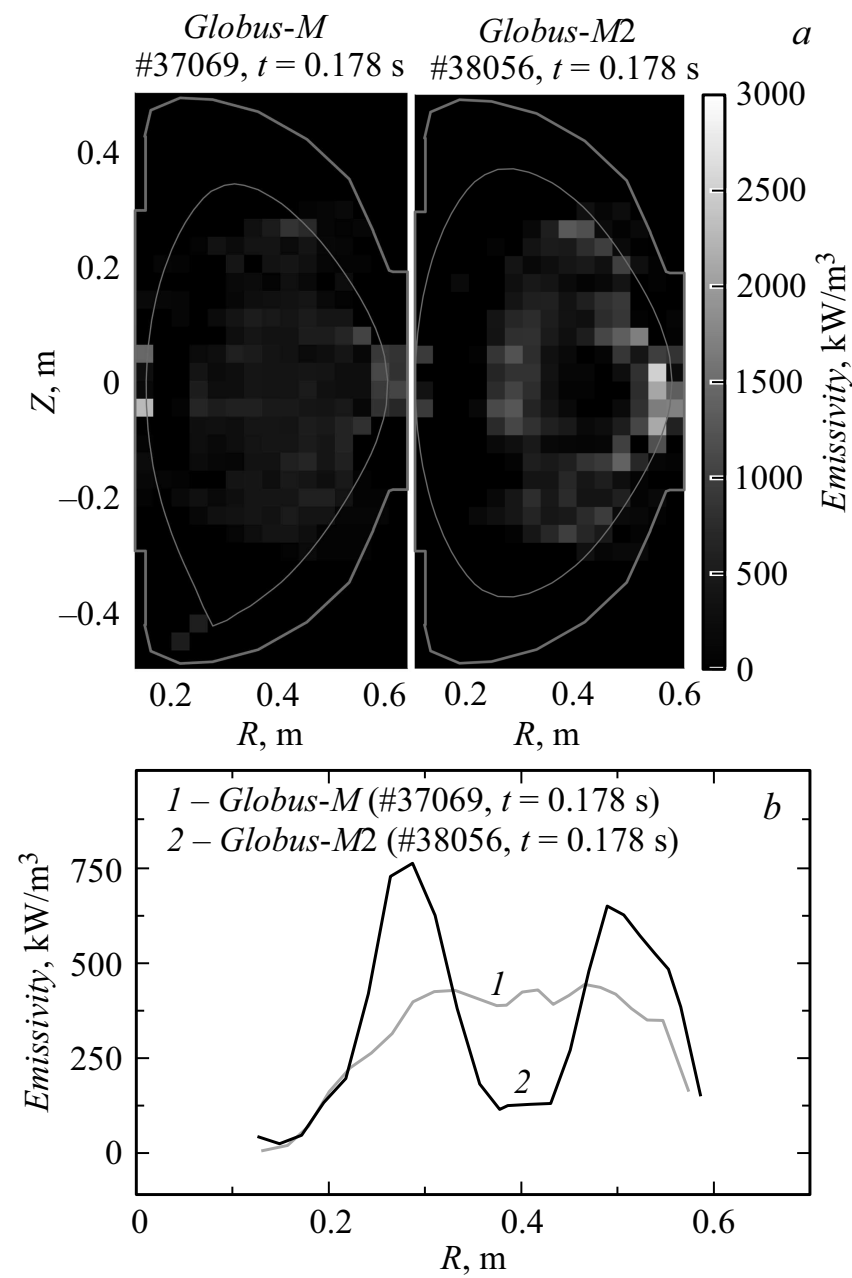

Рис. 2. $a$ - двумерное распределение $P_{\text {rad }}$ в полоидальном сечении с магнитной конфигурацией SOL в разрядах на токамаках Глобус-М при плотности $\left\langle n_{e}\right\rangle=3.62 \cdot 10^{13} \mathrm{~m}^{-3}(\# 37069)$ и Глобус-М2 при плотности $\left\langle n_{e}\right\rangle=3.15 \cdot 10^{19} \mathrm{~m}^{-3}$ (\#38056); $b$ - профили радиационных потерь $P_{r a d}(R)$ в разрядах на токамаках Глобус-М при плотности $\left\langle n_{e}\right\rangle=3.62 \cdot 10^{13} \mathrm{~m}^{-3}(\# 37069)$ (1) и Глобус-М2 при плотности $\left\langle n_{e}\right\rangle=3.15 \cdot 10^{19} \mathrm{~m}^{-3}$ (\#38056) (2).

картина наблюдается на большинстве установок (см., например, зависимость эффективного заряда от электронной плотности, рассмотренную в работах $[8,9])$.

Радиационные потери на токамаке Глобус-М2 измеряют ся с помощью системы на основе фотодиодов SPD (silicon precision detector) [10], состоящей из матрицы $16 \times 16$ и линейки $1 \times 24$, а также дискретных фотодиодов, регистрирующих излучение из различных пространственных областей. Методами томографии восстанавливается двумерное распределение $P_{\text {rad }}$ в полоидальном сечении [11]. Геометрия диагностики была изменена по сравнению с токамаком Глобус-М: линейка была перенесена таким образом, что объемы наблюдения линейки и матрицы пересекались (рис. 1,a). Основной вклад в радиационные потери на токамаке Глобус-М2 вносится линейчатым излучением низкозарядных примесей, по- ступающих со стенки камеры, покрытой графитовыми плитками [11]. Также в экспериментах периодически используется осаждение на поверхность бороуглеродной пленки (боронизация) в тлеющем разряде в смеси гелия и паров карборана [12].

В ходе экспериментальной кампании 2019 г. на токамаке Глобус-М2 были впервые проведены измерения радиационных потерь в разрядах с тороидальным магнитным полем до 0.7 Т и током по плазме до $300 \mathrm{kA}$. Результаты восстановления двумерного распределения $P_{\text {rad }}$ в разряде \#38056 с параметрами $B_{T}=0.7 \mathrm{~T}$, $I_{p}=300 \mathrm{kA}$ представлены на рис. $2, a$, причем мощность полных радиационных потерь из всего объема плазмы $P_{\text {rad tot }}=210 \mathrm{~kW}$, что составило $28 \%$ от мощности нагрева с использованием нейтрального пучка (параметры пучка $\left.P_{\mathrm{NBI}}=850 \mathrm{~kW}, E_{\mathrm{NBI}}=28 \mathrm{keV}\right)$. Мощность нагрева плазмы складывается из двух компонентов: омический нагрев $(522 \mathrm{~kW})$ и поглощенная плазмой мощность из нейтрального пучка $(270 \mathrm{~kW})$. Поглощенная мощность рассчитывается на установке с помощью моделирования взаимодействия пучка с плазмой кодом NUBEAM. Для сравнения здесь также представлен результат измерения $P_{\text {rad }}$ на токамаке Глобус-М для разряда \#37069 с тороидальным магнитным полем $B_{T}=0.4 \mathrm{~T}$ и током по плазме $I_{p}=180 \mathrm{kA}$ (результаты показаны на рис. 2, $a$, параметры - на рис. 2,b), мощность полных радиационных потерь $P_{\text {rad tot }}=170 \mathrm{~kW}$, что составило $43 \%$ от мощности нагрева с использованием нейтрального пучка (параметры пучка $P_{\mathrm{NBI}}=650 \mathrm{~kW}, E_{\mathrm{NBI}}=26 \mathrm{keV}$ ). Мощность омического нагрева составляла $220 \mathrm{~kW}$, мощность нагрева нейтральным пучком - $180 \mathrm{~kW}$. В обоих разрядах присутствует дополнительный нагрев нейтральным пучком, а также разряды имеют приблизительно равные электронную температуру $T_{e}$ и плотность $n_{e}$, однако магнитная конфигурация различается. Двумерное распределение и профили радиационных потерь на рис. 2, $a$ приведены для момента времени $18 \mathrm{~ms}$ от начала инжекции для обоих разрядов.

В разрядах с лимитерной магнитной конфигурацией, в которых проводились измерения, профиль мощности потерь имеет сильный провал в центральной области плазмы. Это может свидетельствовать о том, что на установке Глобус-М2 происходит уменьшение пикированности профиля радиационных потерь: излучение локализовано на периферии плазменного шнура, что является признаком снижения накопления примесей в центральной области плазмы.

Измерения в широком диапазоне электронной плотности плазмы позволили оценить зависимость полных радиационных потерь от плотности электронов. Как видно из рис. 3 , мощность полных радиационных потерь растет с увеличением плотности электронов. Для разрядов с пучком значение $P_{\text {radtot }}$ взято на $18 \mathrm{~ms}$ от начала инжекции, для омических разрядов - в момент времени с максимальной плотностью $n_{e}$ в стационарной стадии разряда. Несмотря на увеличение абсолютной мощности полных радиационных потерь, они составили менее $45 \%$ 


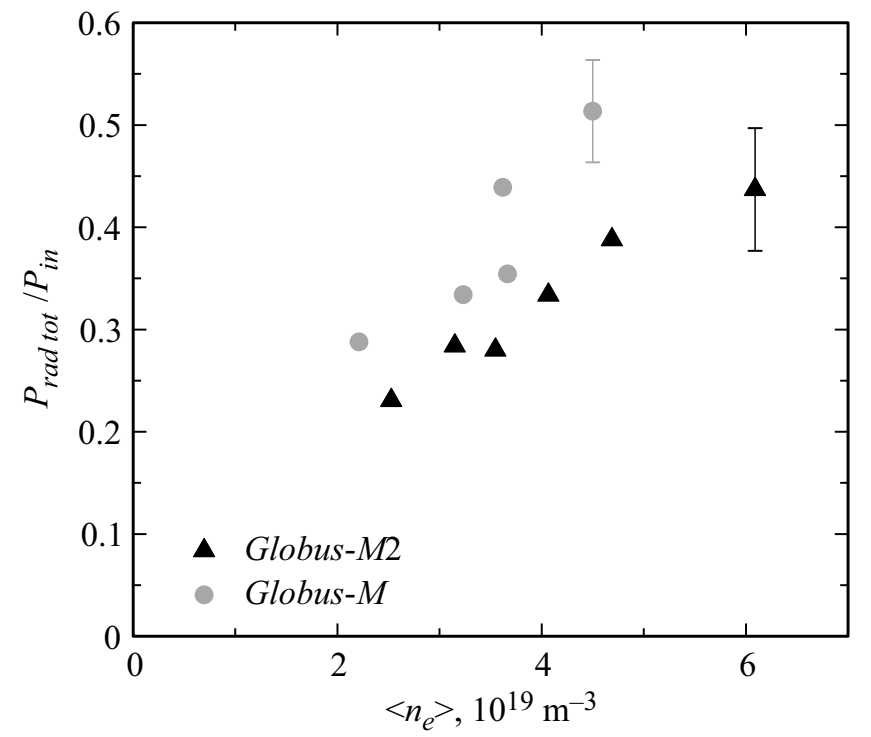

Рис. 3. Зависимость доли радиационных потерь от вкладываемой мощности нагрева $P_{\text {rad tot }} / P_{\text {in }}$ от электронной плотности $\left\langle n_{e}\right\rangle$ на токамаке Глобус-М2.

от вкладываемой мощности. Погрешность $P_{\text {rad }}$ здесь представлена определяемой параметром регуляризации при использовании метода регуляризации Тихонова суммой ошибки восстановления и ошибки из-за шумов. Для разрядов, рассмотренных в работе, погрешность составляла в среднем $10 \%$.

Для сравнения на токамаке ASDEX-Upgrade, камера которого покрыта графитом и вольфрамом, при параметpax плазмы $I_{p}=1 \mathrm{MA}, n_{e}=7 \cdot 10^{19} \mathrm{~m}^{-3}, P_{\mathrm{NBI}}=15 \mathrm{MW}$ мощность радиационных потерь из всего объема плазмы составила 60-80\% от вкладываемой мощности [13].

Таким образом, на токамаке Глобус-М2 в условиях увеличенных значений тороидального магнитного поля $B_{T}$ до $0.7 \mathrm{~T}$ и тока по плазме $I_{p}$ до $300 \mathrm{kA}$ были измерены $\left\langle Z_{e f f}\right\rangle$ в разрядах с различными параметрами плазмы, его временна́я эволюция в течение разряда. Результаты измерения в сериях разрядов позволили получить зависимость $\left\langle Z_{e f f}\left(n_{e}\right)\right\rangle$, которая продемонстрировала уменьшение $\left\langle Z_{e f f}\right\rangle$ с увеличением электронной плотности. Кроме того, в новых условиях модернизированной установки измерены полная мощность радиационных потерь и двумерное распределение мощности радиационных потерь в разрядах при различных $n_{e}$. Экспериментальные результаты, полученные на модернизированной установке Глобус-М2, свидетельствуют об уменьшении доли мощности радиационных потерь от вкладываемой мощности нагрева, а также о возможном уменьшении накопления примесей в центре плазменного шнура (по сравнению с токамаком Глобус-М).

\section{Финансирование работы}

Исследование радиационных потерь выполнено авторами (Е.А. Тюхменева, Г.С. Курскиев, В.Б. Минаев,
Н.В. Сахаров, Е.О. Киселев, А.Ю. Тельнова, Н.Н. Бахарев, П.Б. Щеголев) при финансовой поддержке Российского научного фонда (проект № 17-72-20076).

\section{Конфликт интересов}

Авторы заявляют, что у них нет конфликта интересов.

\section{Список литературы}

[1] V.B. Minaev, V.K. Gusev, N.V. Sakharov, Yu.V. Petrov, V.I. Varfolomeev, N.N. Bakharev, V.A. Belyakov, P.N. Brunkov, V.V. Bulanin, F.V. Chernyshev, V.I. Davydenko, V.V. Dyachenko, A.A. Kavin, S.A. Khitrov, N.A. Khromov, E.O. Kiselev, A.N. Konovalov, V.A. Kornev, G.S. Kurskiev, A.D. Melnik, A.B. Mineev, M.I. Mironov, I.V. Miroshnikov, A.N. Novokhatskii, K.Yu. Oshuev, M.I. Patrov, A.V. Petrov, V.A. Rozhansky, A.N. Saveliev, I.Yu. Senichenkov, P.B. Shchegolev, I.V. Shikhovtsev, A.D. Sladkomedova, V.V. Solokha, D.S. Sorokina, A.Yu. Telnova, V.A. Tokarev, S.Yu. Tolstyakov, A.Yu. Yashin, E.G. Zhilin, J. Phys.: Conf. Ser., 1094, 012001 (2018). DOI: $10.1088 / 1742-6596 / 1094 / 1 / 012001$

[2] V.B. Minaev, V.K. Gusev, N.V. Sakharov, V.I. Varfolomeev, N.N. Bakharev, V.A. Belyakov, E.N. Bondarchuk, P.N. Brunkov, F.V. Chernyshev, V.I Davydenko, V.V. Dyachenko, A.A. Kavin, S.A. Khitrov, N.A. Khromov, E.O. Kiselev, A.N. Konovalov, V.A. Kornev, G.S. Kurskiev, A.N. Labusov, A.D. Melnik, A.B. Mineev, M.I. Mironov, I.V. Miroshnikov, M.I. Patrov, Yu.V. Petrov, V.A. Rozhansky, A.N. Saveliev, I.Yu. Senichenkov, P.B. Shchegolev, O.N. Shcherbinin, I.V. Shikhovtsev, A.D. Sladkomedova, V.V. Solokha, V.N. Tanchuk, A.Yu. Telnova, V.A. Tokarev, S.Yu. Tolstyakov, E.G. Zhilin, Nucl. Fusion, 57, 066047 (2017). https://doi.org/10.1088/1741-4326/aa69e0

[3] V.K. Gusev, N.N. Bakharev, V.A. Belyakov, B.Ya. Ber, E.N. Bondarchuk, V.V. Bulanin, A.S. Bykov, F.V. Chernyshev, E.V. Demina, V.V. Dyachenko, P.R. Goncharov, A.E. Gorodetsky, E.Z. Gusakov, A.D. Iblyaminova, A.A. Ivanov, M.A. Irzak, E.G. Kaveeva, S.A. Khitrov, M.V. Khokhlov, N.A. Khromov, V.V. Kolmogorov, V.A. Kornev, S.V. Krasnov, G.S. Kurskiev, A.N. Labusov, S.A. Lepikhov, N.V. Litunovsky, I.V. Mazul, A.D. Melnik, V.V. Mikov, V.B. Minaev, A.B. Mineev, M.I. Mironov, I.V. Miroshnikov, E.E. Mukhin, A.N. Novokhatsky, A.D. Ovsyannikov, M.I. Patrov, A.V. Petrov, Yu.V. Petrov, V.A. Rozhansky, N.V. Sakharov, A.N. Saveliev, I.Yu. Senichenkov, V.Yu. Sergeev, P.B. Shchegolev, O.N. Shcherbinin, I.V. Shikhovtsev, V.S. Tanaev, V.N. Tanchuk, S.Yu. Tolstyakov, V.I. Varfolomeev, E.O. Vekshina, A.V. Voronin, S.P. Voskoboinikov, F. Wagner, A.Yu. Yashin, G.V. Zadvitskiy, A.P. Zakharov, R.Kh. Zalavutdinov, E.G. Zhilin, Nucl. Fusion., 55, 104016 (2015). DOI: $10.1088 / 0029-5515 / 55 / 10 / 104016)$ 
[4] Н.Н. Бахарев, И.М. Балаченков, В.И. Варфоломеев, А.В. Воронин, В.К. Гусев, В.В. Дьяченко, М.В. Ильясова, Е.О. Киселев, А.Н. Коновалов, Г.С. Курскиев, А.Д. Мельник, В.Б. Минаев, И.В. Мирошников, А.Н. Новохацкий, М.И. Патров, Ю.В. Петров, Н.В. Сахаров, О.М. Скрекель, А.Ю. Тельнова, В.А. Токарев, С.Ю. Толстяков, Е.А. Тюхменева, Е.М. Хилькевич, Н.А. Хромов, Ф.В. Чернышев, И.Н. Чугунов, А.Е. Шевелев, П.Б. Щеголев, Физика плазмы, 46 (7), 579 (2020). DOI: 10.31857/S036729212007001X

[5] J.V. Hofmann, Visible spectroscopy on ASDEX (Garching, Germany, 1991). Report IPP III/174.

[6] E.A. Tukhmeneva, S.Yu. Tolstyakov, G.S. Kurskiev, V.K. Gusev, V.B. Minaev, Yu.V. Petrov, N.V. Sakharov, A.Yu. Telnova, N.N. Bakharev, P.B. Shchegolev, E.O. Kiselev, Plasma Sci. Technol., 21, 105104 (2019). https://doi.org/10.1088/2058-6272/ab305f

[7] Г.С. Курскиев, С.Ю. Толстяков, А.А. Березуцкий, В.К. Гусев, М.М. Кочергин, В.Б. Минаев, Е.Е. Мухин, М.И. Патров, Ю.В. Петров, Н.В. Сахаров, В.В. Семёнов, П.В. Чернаков, ВАНТ. Сер. Термоядерный синтез, № 2, 81 (2012). https://doi.org/10.21517/0202-3822-2012-35-2-81-88

[8] Y. Chen, Z. Wu, W. Gao, L. Zhang, W. Zha, Fusion Eng. Design, 88, 2825 (2013). https://doi.org/10.1016/j.fusengdes.2013.05.003

[9] S. Sarwar, H.K. Na, J.M. Park, Rev. Sci. Instrum., 89, 043504 (2018). https://doi.org/10.1063/1.5004217

[10] A.P. Artyomov, E.H. Baksht, V.F. Tarasenko, A.V. Fedunin, S.A. Chaikovsky, P.N. Aruev, V.V. Zabrodskii, M.V. Petrenko, N.A. Sobolev, V.L. Suhanov, Instrum. Exp. Tech., 58, 102 (2015). https://doi.org/10.1134/S0020441215010017

[11] A.D. Sladkomedova, A.G. Alekseev, N.N. Bakharev, V.K. Gusev, N.A. Khromov, G.S. Kurskiev, V.B. Minaev, M.I. Patrov, Yu.V. Petrov, N.V. Sakharov, P.B. Shchegolev, V.V. Solokha, A.Yu. Telnova, S.Yu. Tolstyakov, V.V. Zabrodsky, Rev. Sci. Instrum., 89, 083509 (2018). https://doi.org/10.1063/1.5039801

[12] V.K. Gusev, T.A. Burtsev, A.V. Dech, G.A. Gavrilov, V.E. Golant, S.V. Krikunov, R.G. Levin, V.B. Minaev, A.B. Mineev, O.A. Minyaev, E.E. Mukhin, A.N. Novokhatskii, Yu.V. Petrov, E.N. Rumyantsev, N.V. Sakharov, V.M. Sharapov, Nucl. Fusion, 41, 919 (2001). https://doi.org/10.1088/0029-5515/41/7/313

[13] J.C. Fuchs, D. Coster, A. Herrmann, A. Kallenbach, K.F. Mast, ASDEX Upgrade Team, J. Nucl. Mater., 290-293, 525 (2001). https://doi.org/10.1016/S0022-3115(00)00645-0 